\title{
Study on the Basic Characteristics of the Wind Turbine Dynamics
}

\author{
Zhiqiang $\mathrm{Xu}$ \\ Department of Physics and Technology, Kunming University, Kunming 650214, China \\ 2793436659@qq.com
}

\begin{abstract}
Keywords: The Horizontal Axis Wind Turbine, The Modal Analysis, The Natural Frequency; Dynamic Characteristics
\end{abstract}

\begin{abstract}
This paper not only analyzes the research status of the wind turbine tower and its infrastructure, but also studies dynamic characteristics of the wind turbine by applying to the finite element analysis. In addition, the tower and the infrastructure are reasonably simplified by adopting the finite element software ANSYS and taking a large section 1.5MW level axis wind turbine for example. The finite element model of tower - base of the horizontal axis wind turbine is established and the modal analysis of the tower-base of the wind turbine is carried on.
\end{abstract}

\section{Introduction}

With the development and utilization of renewable energy of the international community, as a kind of clean energy, wind energy has become a field which many countries develop with each other. In order to increase the output power of the wind turbine, wind turbine is developing in a large scale. Its blades, tower and other structural dimensions are also increasing. For the tower of the wind tower, the basic Kinetic analysis mainly means studying its inherent characteristics, respectively analyzing dynamically of the tower, the base and the tower-base of the wind turbine, resulting in natural frequencies and the vibration modes of the structure. It ensures that the components of the wind turbine will not produce resonance during the operation. On one hand, it ensures the safe operation of the wind turbine. On the other hand, it is of theoretical and practical significance for the optimal design of the wind turbine. The necessary knowledge of the finite element modeling and corresponding numerical analysis theory should be provided so as to analyze the wind turbine dynamic characteristics. Finite element method is developed on the basis of structural mechanics and develops rapidly with the development of the computer. It is a method used to characterize the physical properties of the complex structure, which has been widely used in modern engineering structures. This article describes the basic theory and solving methods of the finite element method and the modal analysis method. Therefore, it has laid a theoretical foundation for the conducting the dynamic characteristics analysis of the wind turbine tower.

\section{Finite Element Method}

The basic idea of FEM is to solve the computational domain into a finite number of non-overlapping units, within each unit, select some appropriate nodes as a function of interpolation points for solving the differential equation variable to rewrite each variable linear expression or a node with the value of the derivative of the selected interpolation function composition. Differential equations are dispersed and soled by means of variation principle or weighted residual method [1]. Adopting different forms of weight functions and interpolation functions constitutes different finite element methods. FEM is most widely used of the numerical calculation methods in today's engineering analysis. The basic idea is [1,2]: the continuous system is divided into a number of finite units, and the equivalent load is introduced on the unit node to take the place of the actual external load; the boundary conditions are introduced, forming a group of unknown variable algebraic equations. Thus the unknown variables at a finite number of nodes will be obtained after it is solved. Finite element method is to idealize a continuous system with an infinite number of degrees of freedom as a set of units with only limited degrees of freedom, converting the problem to the structural problem which is suitable for the numerical solution. As an efficient numerical 
method, the solving process is [1]:

1.Discretization of the structure: the structure will be divided into a finite number of combinations of basic units, then the nodes are provided at each unit and each node adjacent units are connected together to form aggregates, replacing the original structure

2.Characteristic analysis of the unit: the unit displacement is assumed as some kind of simple function coordinates and the node displacement is set to be an unknown quantity. The approximate displacement in the unit is indicated by the node displacement of the unit.

3.Solving the overall stiffness matrix: group the stiffness matrix synthesis of each unit into the overall stiffness matrix and group the equivalent nodal force acting on the node array of each unit into the total load column vector

4.Solving the finite element equation: based on the overall finite element equations of the boundary conditions correction. Solve by using appropriate numerical methods, and the function value of each node can be obtained

\section{Modal Analysis}

Modal means natural vibration characteristics of the structure. The ultimate goal of modal analysis is to identify the modal parameters and provide the basis for the vibration characteristics analysis of the structure, the vibration fault diagnosis and forecasting ,as well as the design optimization of the dynamic characteristics [3]. Theoretically, structure has multi-stage vibration frequency. When the object is in accordance with a natural frequency of vibration, the displacement of each point deviation from the equilibrium position on the object meets certain proportion, which can be represented by a vector. It is called the modal. So the modal order is the order of the corresponding natural frequency. The natural frequencies and the vibration modes of the structure are obtained by using modal analysis. The corresponding kinetic equation is:

$$
[\mathrm{M}]\{\ddot{\mathrm{u}}\}+[\mathrm{K}]\{\mathrm{u}\}=0
$$

The structure is assumed to be linear and response to the harmonic response:

$$
\{\mathrm{u}\}=\left\{\phi_{i}\right\} \cos \left(\omega_{j}\right)
$$

In the formula, $\phi_{i}$-the vibration mode, $\omega_{j}$-the natural frequency

Formula (1) into (2), there is:

$$
\left(\mathrm{K}-\omega_{i}^{2}[\mathrm{M}]\right)\left\{\phi_{i}\right\}=0
$$

By solving the formula (3) vibration mode and $\phi_{i}$ natural frequency $\omega_{j}$ can be obtained.

Table 1 The first six stages of the natural frequency of the base $(\mathrm{Hz})$

\begin{tabular}{|c|c|c|c|c|c|c|}
\hline Frequency stage & 1 & 2 & 3 & 4 & 5 & 6 \\
\hline Base model 1 & 207.98 & 208.25 & 250.79 & 250.96 & 356.44 & 356.65 \\
\hline Base model 2 & 2.765 & 2.765 & 3.351 & 3.634 & 10.592 & 10.778 \\
\hline Base model 3 & 2.501 & 2.501 & 3.316 & 3.426 & 10.461 & 10.630 \\
\hline
\end{tabular}




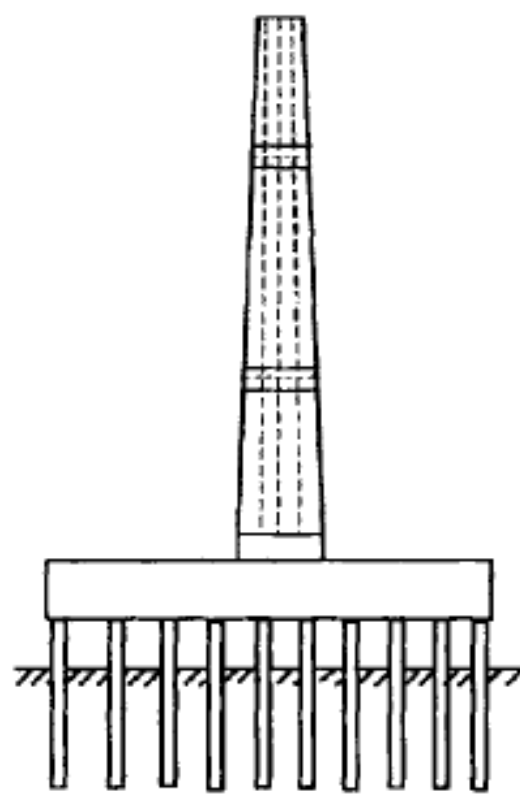

Fig.1The tower-base model schematic diagram based on more concrete piles bearing desktop

For the tower infrastructure of the wind turbine studied in this paper, there are three meaningful vibration modes when its dynamic characteristics are calculated, namely, lateral bending vibration mode, back and forth bending vibration mode and rotational vibration mode [4]Therefore, the modal analysis need be conducted when the tower is designed in order to understand its dynamic characteristics, from which it can be determined whether the natural frequency of the tower will coincide with the frequency of rotation of the impeller, or whether it avoids a certain range of the rotation of the impeller excitation frequency [5,6]. After the base- tower modal has been analyzed and the effects of changes in the underlying pile stiffness in different directions on the natural frequency of the wind turbine has been studied, the horizontal stiffness of the pile has a greater effect on a higher order frequency than on a lower order frequency; the vertical stiffness has a significant effect on different frequencies. When the pile stiffness reaches to a certain value rigidity, the impact on the frequency levels off. The changes in quality of the wind turbine head has an obvious influence on the natural frequency changes of the tower, especially on a lower order frequency of the wind turbine. When the head mass increases to a certain weight, the effect on the frequency gradually slows down. The responses at different locations of the tower at different excitation frequencies can be obtained after the tower harmonic response is analyzed. The vibration of base-tower takes place mainly with lower frequency, especially for the vibration amplitude of the tower in the direction of wind loads, which is consistent with the actual working conditions of the wind turbine. It provides a theoretical basis for predicting the dynamic characteristics of the wind turbine structures, analyzing the resonance, fatigue and other harmful effects produced by forced vibration..

\section{Conclusion}

Based on the analysis of the mechanical theory as the foundation, designed the soccer robot pick the ball institutions optimal design process, found aim function, select design variables and the corresponding optimization algorithm to optimize a complete set of institutions. At last through the test to get the final performance parameters of the institution. Experiments show that the system has higher accuracy and stability, the new optimize pick the ball have design basic requirements, and achieved good ideal control effect.

Establish the base and the overall tower models of the tower-base, analyze the dynamic characteristics by using the finite element software ANSYS, and relevant results will be obtained. The basic natural frequencies and mode shapes can be obtained through infrastructure modal analysis. The natural frequency values are far higher than the rotor excitation frequency and the natural frequency of the tower, avoiding the resonance of the wind turbine. The first and second 
order vibration modes of the base belong to the horizontal side vibration. The third is rotating vibration around the axis. The fourth vibrates in the vertical direction. The interaction between the tower and the base reduces the natural frequency of the tower. Different stiffness has different effect on the dynamic characteristics. The basic level of stiffness has less influence on the overall lower order frequency, while greater on higher order frequency; vertical stiffness and rotational stiffness have more significant impact on the overall natural vibration frequency of the tower-base.

\section{References}

[1] Xucheng Wang. Finite Element Method [M]. Beijing: Tsinghua University Press, 2003,5-7.

[2] Renxian Li. Basic Finite Element Method [M]. Beijing: National Defense Industry Press, 2004, 3-6.

[3] Zhenhua Ni. Vibration Mechanics [M]. Xi'an: Xi'an Jiaotong University Press, 1989.

[4] Benli Li, Xiangeng Song. Wind Turbine Structural Dynamics [M]. Beijing: Beijing Aerospace University Press, 1999.

[5] Lixin Zhao. The Finite Element Analysis and Optimization of the Wind Turbine Tower Design [D]. [Jilin University Master's Degree Theses]. Jilin: Jilin University, 2008.

[6] Yinguang Wu. Rigid Coupling Dynamic Analysis to Large Horizontal Axis Wind Turbine [D]. [Master's Degree Theses of Lanzhou University] Lanzhou: Lanzhou University of Science and Engineering, 2013: 26-27. 